\title{
EPITHELIAL INLAY IN RADICAL MASTOID OPERATIONS.*
}

By E. HAMILTON WHITE, Montreal.

THE idea of skin grafting the bone cavity after a radical mastoid operation has long been advocated by Sir Charles Ballance. While the advantages have been recognised, it has never been widely popular owing to the difficulty of technique and uncertainty of result.

By the use of the present technique the operation is made extremely simple and rapid, and the success of the graft is practically assured. The method is an application of Esser's idea of epithelial inlay to the local surgical needs of a radical mastoid operation. The idea of using it was suggested to me last summer by Major E. F. Risdon, C.A.M.C., who had used it widely in his plastic surgery about the face and mouth. The present paper is really a preliminary report, further cases will be reported later by Major Risdon and Lieut.-Colonel Perry Goldsmith, of Toronto.

The question as to whether the skin grafting should be done at the time of the primary operation or as a secondary operation, ten days or so later, is not important for the present report, which is concerned only with the technique of the grafting, which is the same for all cases. Where the skin grafting is done as a secondary operation the plastic should be postponed and done at the same time. In both my cases the grafting was done as a secondary operation for the reasons stated in the reports.

The cavity is prepared in the ordinary way by curetting and flushing with warm saline. The wound being ready for grafting, an exact cast of the bone cavity is made by pressing into it a piece of dental compound (stent). The stent is softened by immersion in hot water, but the water must not be boiling or it renders the compound too soft and sticky. As soon as it hardens the cast is withdrawn from the wound, a skin graft is laid over it with the cut surface outward, and it is then pushed back into position.

* Reported before the Montreal Medico-Chirurgical Society, and December $192 \mathrm{I}$. 


\section{E. Hamilton White}

This simple technique insures an even distribution and close contact of the graft with the walls of the cavity. The close contact with the wound surface insures good nourishment, and prevents the collection of serum between the walls of the cavity and the graft which would tend to displace the graft, and would also predispose to infection. The dental compound being nonabsorbent, there is no discharge soaked dressing to encourage bacterial growth in the wound.

A further advantage of the technique is that the flaps of the plastic are held firmly in good position, and rapid healing encouraged.

The cast remains in position a week, unless some untoward circumstance calls for its removal. In my cases it was removed through the retro-auricular opening, as it was felt that an attempt to remove it through the enlarged meatus was adding unnecessary difficulty to the operation, and the delay caused by keeping the wound open a week is trifling, as upon removal of the cast it heals rapidly without suture.

The great advantage of a skin graft following a radical mastoid operation is the rapidity of healing. Another thing gained by skin grafting, if successful, is that the walls of the bone cavity are brought to rest, and the tendency to exuberant granulations and cicatricial narrowing of the cavity is done away with. The tendency to unhealthy granulation is especially associated with malnutrition of the patient.

The two cases in which the method was used are of some interest, and were as follows:-

CASE I.-W. S., female, aged $2 \mathbf{I}$. Admitted to Royal Victoria Hospital, $29^{\text {th }}$ June $192 \mathrm{l}$, with a history of active discharge from the right ear for two years without earache, but for the last few months with headache. The origin of the suppuration was obscure, dating back to childhood.

The patient is a cripple from old bip-joint and spinal disease, but at present these lesions are healed and she looks fairly healthy. There is no evidence of any active trouble in the ear.

The right ear showed a destruction of the posterior half of the drumhead and a moderately free purulent discharge from the attic. The functional tests showed doubtful remains of hearing, only loud voice at the ear, but the vestibular reactions were present indicating an intact labyrinth.

Radical Mastoid Operation, i ith July.-At the operation the mastoid showed a chronic abscess in the region of the antrum and 


\section{Epithelial Radical Mastoid Operations}

body of the mastoid. The cavity was filled with granulations, but there was no evidence of cholesteatomatous matrix. In removing the overhanging edges of the cavity the dura was exposed to a considerable extent in the region of the middle fossa. For this reason the cavity was left open and allowed to granulate until zoth July when the plastic and skin graft were undertaken. The cavity was curetted and grafted as above described.

No pain or discomfort was experienced after the operation, and the cast was removed one week later without an anæsthetic. On removal of the cast the cavity was beautifully lined with skin which remained healthy and showed the complete success of the skin graft.

In this case the graft was cut from the side of the calf, as owing to her crippled condition it proved the easiest area from which to procure the graft.

The patient was discharged on $13^{\text {th }}$ August, approximately three weeks after grafting the cavity, at that time being well healed. The case has been under observation since, and has remained permanently dry.

Case II.-M. W., female, aged 42. Was admitted to the Royal Victoria Hospital 22 nd July I 92 I, complaining of dizziness, vomiting, and discharge from the left ear. The history was of an attack of pain in the left ear three weeks ago, followed by discharge which gave relief. The discharge stopped after a day or two, and the patient felt fairly well. She then noticed the onset of dizziness on walking or standing, and this was associated with vomiting. The dizziness was always associated with movement, and has disappeared while lying in bed.

The history of the original onset of the trouble in the ear could not be obtained, but the suppuration was evidently a matter of old standing though apparently quite latent for many years. The hearing in the left ear was relatively good, conversation voice at 6 or 8 feet. There was a perforation of the posterior upper quadrant of the drumhead, and a small amount of purulent discharge with foul odour. There was a spontaneous nystagmus to the left, unsteadiness on standing, and a tendency to fall to the right. The right ear was normal.

On applying the fistula test, although only gentle compression was used, a violent reaction was produced, the patient throwing her body to the right. A diagnosis of a chronic mastoiditis with a labyrinth fistula was made. Dr Russel our neurologist agreed, and reported no evidence of intracranial involvement.

Radical Mastoid Operation, 27 th July.-At the operation a small antrum was found, deeply placed, and filled with cholesteatoma, and a large fistula was readily seen in the lateral semi-circular canal. 


\section{E. Hamilton White}

Owing to the small deeply placed antrum an extensive exposure of the middle fossa occurred with the removal of the overhanging edges. In this case a primary skin graft would have been definitely dangerous owing to the presence of the fistula.

The wound was allowed to granulate for three weeks, and then grafted as in the other case; the plastic being done at the same time as the graft on 17 th August. Only a small graft was required, and this was taken from the front of the thigh. The operation was followed by no undue reaction, and the cast was removed in a week. The patient was discharged on 7 th September, well healed, approximately three weeks after the application of the skin graft. 